\title{
THE SINGULAR DIRICHLET PROBLEM FOR THE COMPLEX MONGE-AMPÈRE OPERATOR ON COMPLEX MANIFOLDS
}

\author{
By Makoto SUzUKI
}

\section{Introduction.}

Let $M$ be a connected paracompact complex manifold of dimension $n$ with a fixed volume form $d V$, and $\Omega$ a relatively compact, strictly pseudoconvex open subset of $M$. Bedford and Taylor [3] showed that

$$
\left(d d^{c} u\right)^{n}:=\wedge^{n} d d^{c} u
$$

is well defined as a positive Radon measure for a locally bounded, plurisubharmonic function $u$ on $\Omega$, where $d^{c}=\sqrt{-1}(\bar{\partial}-\partial)$. We call the assignment $u \rightarrow$ $\left(d d^{c} u\right)^{n}$ the complex Monge-Ampère operator. In this paper we study the nonlinear $(n>1)$ Dirichlet problem for the complex Monge-Ampère operator:

$$
\begin{aligned}
& u \text { is plurisubharmonic on } \Omega, \\
& \lim _{z \rightarrow \partial \Omega} u(z)=\phi \quad \text { on } \partial \Omega, \\
& \left(d d^{c} u\right)^{n}=F(u, z) d V \quad \text { on } \Omega,
\end{aligned}
$$

where $\phi$ is a real-valued function on $\partial \Omega$ and $F$ a non-negative function on $\Omega$. Many results on the existence and the regularity of the solution of (1.1) were obtained in [1], [3], [4], [5], [7], [8], [9], [11], [12], [13], etc. In the case of singular boundary data (i. e., $\phi=+\infty$ ), however, the singular Dirichlet problem seems to be unknown except for some special cases (for example, [5], [10], [14]), some of which are treated in the context of the existence of the complete KählerEinstein metric. We will show the existence of generalized solution of the equation (1.1) for the singular boundary data on Stein manifold, and extend Theorem 5 in Bedford and Taylor [5], which states that if $\Omega$ is a bounded strictly pseudoconvex set in $\boldsymbol{C}^{2}, F \in C(\boldsymbol{R} \times \bar{\Omega}), F \geqq 0, t \rightarrow F(t, z)$ increasing in $t$, $t \rightarrow[F(t, z)]^{1 / 2}$ a convex function of $t$, and $F$ has an upper barrier, then $u(z):=$ $\sup \left\{v(z): v \in P(\Omega) \cap L_{\mathrm{loc}}^{\infty}(\Omega), \Phi(v) \geqq[F(t, z)]^{1 / 2}\right\}$ is a solution of $(1.1)$ with $\phi=+\infty$. As a result we will establish

THEOREM. Let $M$ be an $n$-dimensional Stern manfold with volume form $d V$ and $\Omega$ a relatively compact, $C^{0}$ strictly pseudoconvex domain in $M$. Let $F \in L_{\mathrm{loc}}^{\infty}$

Received September 26, 1988 
$(\boldsymbol{R} \times \Omega)$ be a positive function such that $F(t, z) \geqq e^{k t}$ on $\boldsymbol{R} \times \Omega$ for some constant $k>0, F(t, z)$ is bounded on $\Omega$ for each $t \in R$ fixed, and continuous, nondecreasing in $t$ for every fixed $z \in \Omega$. Then the upper envelope

$$
u(z):=\sup \left\{v(z) ; v \in P(\Omega) \cap L_{\mathrm{loc}}^{\infty}(\Omega),\left(d d^{c} v\right)^{n} \geqq F(v, z) d V\right\}
$$

is a maximal solution of the Dirichlet problem:

$$
\begin{aligned}
& u \in P(\Omega) \cap L_{\mathrm{loc}}^{\infty}(\Omega), \\
& \lim _{\Omega \ni z \rightarrow \zeta} u(z)=+\infty \quad \text { for } \zeta \in \partial \Omega, \\
& \left(d d^{c} u\right)^{n}=F(u, z) d V \quad \text { on } \Omega .
\end{aligned}
$$

In $\S 2$, we construct the solution of (1.1) on Stein manifolds in the case that $\phi<+\infty$. In $\S 3$, we consider the singular Dirichlet problem (Theorem).

The author would like to express his sincere gratitude to Professor J. Noguchi for his encouragement and many suggestions and to Professor E. Bedford for valuable advice.

Definitions and notation. The class of plurisubharmonic functions on $M$ is denoted by $P(M)$. A plurisubharmonic function $u$ defined on $M$ is called strictly plurisubharmonic, if $u$ is plurisubharmonic on $M$ and if for an arbitrary complex local coordinate system $z=\left(z^{1}, \cdots, z^{n}\right)$, there exists some constant $\delta>0$ such that $u-\delta\|z\|^{2}$ is also plurisubharmonic on the chart where $\|z\|$ denotes the usual Euclidean norm in $\boldsymbol{C}^{n} \cong \boldsymbol{R}^{2 n}$.

An open set $\Omega$ in a complex manifold is said to be $C^{k}$ strictly pseudoconvex ( $k \geqq 0$ is some integer) if for each $\zeta \in \partial \Omega$ there exist a neighborhood $N$ of $\zeta$ and a $C^{k}$ strictly plurisubharmonic function $\rho$ on $N$ such that

$$
N \cap \Omega=\{z \in N ; \rho(z)<0\}, \quad N \cap \partial \Omega=\{z \in N ; \rho(z)=0\},
$$

and in case of $k \geqq 1 d \rho \neq 0$ on $N \cap \partial \Omega$.

\section{Existence of generalized solutions for nonsingular case.}

Let $M$ be an $n$-dimensional complex manifold equipped with a hermitian metric and a volume form $d V$, and let $\Omega$ be an open subset in $M$. For a realvalued function $\phi$ on $\partial \Omega$ and a (Lebesgue) measurable function $f$ on $\bar{\Omega}$ (the closure of $\Omega$ ), we define the following Perron family

$$
\begin{aligned}
L(\phi, f):= & \left\{v \in P(\Omega) \cap L_{\mathrm{loc}}^{\infty}(\Omega) ; \lim _{\Omega \ni z \rightarrow \zeta} v(z) \leqq \phi(\zeta), \zeta \in \partial \Omega,\right. \\
& \text { and } \left.\left(d d^{c} v\right)^{n} \geqq f d V \text { on } \Omega\right\} .
\end{aligned}
$$

We denote by $u^{*}$ the upper regularization of $u$ : i. e., $u^{*}(z)=\limsup _{\Omega \ni x \rightarrow z} u(x)$. By making use of $L(\phi, f)$ as in Bremermann [7; Theorem 4.1] and Bedford-Taylor [3; Theorem 6.2$]$, we have the following 
Proposition 2.1. Let $\phi \in C(\partial \Omega), f \in L^{\infty}(\Omega)$ with $f \geqq 0$. Suppose that $\Omega$ is $C^{0}$ strictly pseudoconvex and $L(\phi, f)$ is not empty. Then the upper envelope

$$
u(z):=\sup \{v(z) ; v \in L(\phi, f)\}, \quad z \in \Omega
$$

satısfies that $\lim _{\Omega \ni z \rightarrow \zeta} u(z)=\phi(\zeta)$ for $\zeta \in \partial \Omega$, and $u \in L(\phi, f)$.

Remark. If there exists a $C^{0}$ strictly plurisubharmonic function on $\Omega$, then the above $L(\phi, f)$ is not empty and we can consider $\Omega$ to be a $C^{0}$ strictly pseudoconvex domain in a Stein manifold by Grauert's theorem.

Proposition 2.1 shows that the upper envelope is necessarily continuous at the boundary of $\Omega$ if the boundary data $\phi$ is continuous. The argument due to Walsh [16] is useful to show the continuity of the upper envelope in the whole of $\Omega$. Applying the argument as in Bedford-Taylor [3; Theorem 6.2], Bedford [2] and Asaba [1] (see also Kaneko [13]), we obtain

THEOREM 2.2 Let $M$ be an n-dimensional Stein manfold with a fixed volume form $d V$, and $\Omega$ be a $C^{0}$ strictly pseudoconvex, relatively compact domain in $M$. If $\phi \in C(\partial \Omega)$, and $f \in C(\bar{\Omega})$ is a bounded nonnegative function, then the upper envelope

$$
u(z):=\sup \{v(z) ; v \in L(\phi, f)\}, \quad z \in \Omega
$$

is continuous on $\Omega$ and is extended continuously to $\bar{\Omega}$.

Now we will construct the generalized solutions for the Dirichlet problem on complex manifold. In the case that $M=C^{n}$ with the usual Kähler metric, Cegrell [11; Lemma 2] showed that if $\Omega$ is a strictly pseudoconvex domain in $C^{n}, \phi \in C(\partial \Omega)$ and $f \in L^{\infty}(\Omega), f \geqq 0$, then the Dirichlet problem for the complex Monge-Ampère operator :

$$
\begin{aligned}
& u \in P(\Omega) \cap L^{\infty}(\Omega), \\
& \lim _{\Omega \ni z \rightarrow \zeta} u(z)=\phi(\zeta) \quad \text { for } \quad \zeta \in \partial \Omega, \\
& \left(d d^{c} u\right)^{n}=f d V \quad \text { on } \Omega,
\end{aligned}
$$

has a unique solution, where $d V$ is $2 n$-dimensional Lebesgue measure. Thus Proposition 2.1 and the minimum principle in Bedford and Taylor [6; Corollary 4.4] (see also Lemma 3.2 below) yield

THEOREM 2.3. Let $\Omega$ be as above. Let $\phi \in C(\partial \Omega)$ and $f \in L^{\infty}(\Omega)$ with $f \geqq 0$. Then the upper envelope $u$ of $L(\phi, f)$ is a unique solution of the Dirichlet problem (2.1).

This was also proved by Fukushima [11; Corollary 1], using the method of spherical modification with the extended versions of the minimum principle $[11$; Theorem 4 and 5], which were obtained by the stochastic method. The method 
in Fukushima is valid in our case on general complex manifolds. We shall obtain the following lemma employing the method, but without Theorem 4 and 5 in [11]. For the sake of completeness, we present here an elementary proof.

LEMMA 2.4. Let $M$ be an n-dimensional complex manifold equipped with a volume form $d V$, and $\Omega$ be an open subset in $M$. Suppose that $\phi$ is a real-valued (admitting values $\pm \infty$ ) function on $\partial \Omega$, and $f \in L_{\mathrm{loc}}^{\infty}(\Omega)$ with $f \geqq 0$. If $L(\phi, f)$ is not empty and if the upper envelope

$$
u(z):=\sup \{v(z) ; v \in L(\phi, f)\}, \quad z \in \Omega
$$

is locally bounded, then $\left(d d^{c} u^{*}\right)^{n}=f d V$ on $\Omega$.

Proof. We first show that for each $z \in \Omega$, there exists a neighborhood $N \subset \Omega$ such that for any $v \in L(\phi, f)$ there exists a plurisubharmonic function $\tilde{v} \in L(\phi, F)$ satisfying the following conditions:

(1) $\tilde{v} \geqq v$ on $\Omega$,

(2) $\tilde{v}=v$ of $\Omega \backslash N$,

(3) $\left(d d^{c} \tilde{v}\right)^{n}=f d V$ on $N$.

Further, if $v_{1}, v_{2} \in L(\phi, f)$ and $v_{1} \leqq v_{2}$, then $\tilde{v}_{1} \leqq \tilde{v}_{2}$ where $\tilde{v}_{j}$ is the above modification of $v_{\jmath}, j=1,2$. Let $B$ and $N$ be two neighborhoods of $z$ in one same local chart around $z$ with $\Omega \fallingdotseq B \supseteq N$, and $N$ be biholomorphic to a ball in $\boldsymbol{C}^{n}$. Let $v_{j} \in L(\phi, f), j=1,2$ with $v_{1} \leqq v_{2}$ and for $\varepsilon>0, v_{j \varepsilon}=v_{j} * \chi_{\varepsilon}$ be the smoothing of $v_{j}$ on $B$, where $\chi_{\varepsilon}$ is a usual smoothing kernel. Then $v_{\rho \varepsilon}$ is a smooth plurisubharmonic function in a neighborhood of $\bar{N}, v_{1 \varepsilon} \leqq v_{2 \varepsilon}$ on $\bar{N}$ for $\varepsilon>0$ and $v_{9 \varepsilon} \rightarrow v$, on $\bar{N}$ decreasingly as $\varepsilon \rightarrow 0$. By Theorem 2.3, for each $j$ and $\varepsilon>0$ there exists a plurisubharmonic function $w_{\jmath \varepsilon}$ on $\bar{N}$ such that $\left(d d^{c} w_{\jmath \varepsilon}\right)^{n}=f d V$ on $N$ and $w_{\jmath \varepsilon}=v_{\rho \varepsilon}$ on $\partial N$. It follows from the minimum principle (cf. Bedford-Taylor [6; Corollary 4.4]) that $w_{1 \varepsilon} \leqq w_{2 \varepsilon}$ on $\bar{N}$ and $w_{\jmath \varepsilon}$ decreases as $\varepsilon \rightarrow 0$ for $j=1,2$. Since $v_{\jmath} \in$ $L\left(v_{\jmath \varepsilon} \mid \partial N, f\right)$, we have $v_{\jmath} \leqq w_{\jmath \varepsilon}$ on $N$. Thus the function $w_{j}(z):=\lim _{\varepsilon} w_{\jmath \varepsilon}(z)(z \in N)$ is bounded plurisubharmonic on $N$ for $j=1,2$ and we have $w_{1} \leqq w_{2}$ on $N$. Put

$$
\tilde{v}_{j}(z):= \begin{cases}w_{j}(z) & z \in N \\ v_{j}(z) & z \in \Omega \backslash N .\end{cases}
$$

Then $\tilde{v}_{j} \in P(\Omega) \cap L_{\text {loc }}^{\infty}(\Omega)$ for $j=1,2$ and $\tilde{v}_{1} \leqq \tilde{v}_{2}$ on $\Omega$. Since $\left(d d^{c} \tilde{v}_{j}\right)^{n}=f d V$ on $N$ and the volume of $\partial B$ is zero, we have $\left(d d^{c} \tilde{v}_{j}\right)^{n} \geqq f d V$ on $\Omega$. Since $\tilde{v}_{j}=v$, near $\partial \Omega, \tilde{v}_{j} \in L(\phi, f)$ and $\tilde{v}$, satisfies conditions (1), (2) and (3). By Choque's Lemma we can choose a sequence $\left\{u_{j}\right\} \subset L(\phi, f)$ with $u_{1} \leqq u_{2} \leqq \cdots$ and $u_{j} \rightarrow u^{*}$ almost everywhere $(d V)$ in $\Omega$ (see Lelong [15; p 26]). Since the result is local, it is sufficient to prove in a neighborhood of each $z \in \Omega$. Let $z \in \Omega$ be fixed. Take

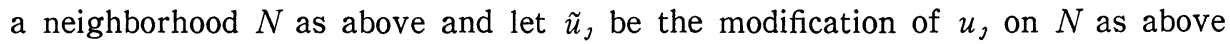
for each $j$, i. e., $\tilde{u}_{j} \in L(\phi, f), \tilde{u}_{j} \geqq u$, on $\Omega, \tilde{u}_{j}=u$, on $\Omega \backslash N$ and $\left(d d^{c} \tilde{u}_{j}\right)^{n}=f d V$ on $N$. Then from the above fact, if $j<k$, we have $\tilde{u}_{\jmath} \leqq \tilde{u}_{k}$ on $\Omega$. On the other 
hand, since $u_{\jmath} \leqq \tilde{u}_{\jmath} \leqq u \leqq u^{*}$ on $\Omega$, we have $\lim \tilde{u}_{j}(z)=u^{*}(z)$ almost everywhere on $\Omega$. If $u$ is locally bounded, so is $u^{*}$, it then follows from the continuity of the operator $\left(d d^{c}\right)^{n}$ (cf. Bedford-Taylor [6; Proposition 5.2]) that $\left(d d^{c} u^{*}\right)^{n}=\lim _{j}\left(d d^{c} \tilde{u}_{j}\right)^{n}$ $=f d V$ on $N$.

Q.E.D.

From the above proposition and Proposition 2.1, we obtain

THEOREM 2.5. Let $M$ be an n-dimensional complex manifold with a volume form $d V$ and $\Omega$ a $C^{0}$ strictly pseudoconvex, relatively compact domain in $M$. Let $\phi \in C(\partial \Omega)$ and $f \in L^{\infty}(\Omega)$ with $f \geqq 0$. Then if $L(\phi, f)$ is not einpty, the upper envelope of $L(\phi, f)$

$$
u(z):=\sup \{v(z) ; v \in L(\phi, f)\}, \quad z \in \Omega
$$

is a solution of the Diruchlet problem:

$$
\begin{aligned}
& u \in P(\Omega) \cap L^{\infty}(\Omega) \\
& \lim _{\Omega \ni z \rightarrow \zeta} u(z)=\phi(\zeta) \quad \text { for } \quad \zeta \in \partial \Omega, \\
& \left(d d^{c} u\right)^{n}=f d V \quad \text { on } \Omega .
\end{aligned}
$$

Moreover, if there exists a locally bounded strictly plurisubharmonic function on $\Omega$, the above $u$ is in fact, the unique solution of the Dirichlet problem (2.2).

\section{Singular Dirichlet Problem.}

We consider the following singular Dirichlet problem

$$
\begin{aligned}
& u \in P(\Omega) \cap L_{\mathrm{loc}}^{\infty}(\Omega), \\
& \lim _{\Omega \ni z \rightarrow \zeta} u(z) \equiv+\infty \quad \text { for } \quad \zeta \in \partial \Omega, \\
& \left(d d^{c} u\right)^{n}=F(u, z) d V \quad \text { on } \Omega,
\end{aligned}
$$

where $F(t, z) \in L_{\mathrm{loc}}^{\infty}(R \times \Omega)$ be a nonnegative function. By using the continuous dependence of the solution on the domain and the data, we are able to obtain the solutions of equation (3.1) under additional assumptions on $F$. In order to utilize the continuous dependence of the solution on the data, we first solve the Dirichlet problem (3.1) for the continuous boundary data. By combining the same technique as used in Cegrell [9] with our result of Theorem 2.5, we obtain

THEOREM 3.1. Let $M$ be an n-dimensional Stein manifold with a volume form $d V$, and $\Omega$ a $C^{0}$ stractly pseudoconvex, relatively compact domain in $M$. Let $\phi \in$ $C(\partial \Omega), F \in L^{\infty}((-\infty, \max \phi] \times \Omega)$ with $F \geqq 0$, and $F(t, z)$ be continuous in $t$ for every fixed $z \in \Omega$. Then there exists a solution of the Dirichlet problem 


$$
\begin{aligned}
& u \in P(\Omega) \cap L^{\infty}(\Omega), \\
& \lim _{\Omega \ni z \rightarrow \zeta} u(z)=\phi(\zeta) \quad \text { for } \quad \zeta \in \partial \Omega, \\
& \left(d d^{c} u\right)^{n}=F(u, z) d V \quad \text { on } \Omega .
\end{aligned}
$$

In general, the solution of (3.2) is not unique (see Cegrell [9]). Assuming an aditional condition, we have the uniqueness by the following minimum principle:

LEMMA 3.2. Let $M$ be an $n$-dimensional complex manfold, and $\Omega$ be a relatively compact open subset in $M$ with a globally defined, locally bounded strictly plurisubharmonic function. Let $F(t, z)$ be a nonnegative function on $\boldsymbol{R} \times \Omega$ such that $F(t, z)$ is nondecreasing in $t$ for every fixed $z \in \Omega$. If $u_{1}, u_{2} \in P(\Omega) \cap L^{\infty}(\Omega)$ satisfy

$$
\begin{array}{ll}
\liminf _{\Omega \ni z \rightarrow \zeta}\left(u_{1}(z)-u_{2}(z)\right) \geqq 0 & \text { for } \zeta \in \partial \Omega, \\
\left(d d^{c} u_{1}\right)^{n} \leqq F\left(u_{1}, z\right) d V & \text { on } \Omega, \\
\left(d d^{c} u_{2}\right)^{n} \geqq F\left(u_{2}, z\right) d V & \text { on } \Omega,
\end{array}
$$

then $u_{1} \geqq u_{2}$ on $\Omega$.

The proof is carried out by only miner modifications of the proof of Corollary 4.4 in Bedford and Taylor [6] by using the comparison theorem, Theorem 4.3 of Bedford [2].

By the above lemma, we see that if $t \rightarrow F(t, z)$ is nondecreasing for every fixed $z \in \Omega$ in Theorem 3.1, the solution of the problem (3.2) is unique; then the solution is the upper envelope of $L(\phi, F):=\left\{v \in P(\Omega) \cap L_{1 \mathrm{loc}}^{\infty}(\Omega) ;\left(d d^{c} v\right)^{n} \geqq F(v, z) d V\right.$, $\limsup _{\Omega \ni z \rightarrow \zeta} v(z) \leqq \phi(\zeta)$ for $\left.\zeta \in \partial \Omega\right\}$.

We need the following notion due to Bedford and Taylor [5; p. 47].

Definition. A function $\beta(z)$ locally bounded from above on $\Omega$ is said to be an upper barrier for $F(t, z)$, if $\beta \geqq v$ on $\Omega$ for all $v \in P(\Omega) \cap L_{\mathrm{loc}}^{\infty}(\Omega)$ with $\left(d d^{c} v\right)^{n}$ $\geqq F(v, z) d V$ on $\Omega$.

We give a weak criterion for the existence of the upper barrier for given $F$, which implies the singular Dirichlet problem (3.1) is solvable if it is locally solvable. Using Lemma 3.2 we have

LEMMA 3.3. Let $M$ be an n-dimensional complex manifold with a volume form $d V, \Omega$ a relatively compact open subset in $M$, and $F(t, z) \in L_{\mathrm{loc}}^{1}(\boldsymbol{R} \times \Omega)$ a nonnegative function. If for each $z \in \Omega$, there exist a neighborhood $N$ of $z$ in $\Omega$ and an upper barrier for $F$ on $N$, then $F$ has an upper barrier on $\Omega$. In particular, if for each $z \in \Omega$ there exists a strictly pseudoconvex, relatively compact neighborhood $N$ of $z$ in $\Omega$ and a function $\beta \in P(N) \cap L_{\mathrm{loc}}^{\infty}(N)$ such that $\beta(z) \rightarrow+\infty$ as $N \ni z \rightarrow \partial N$ and $\left(d d^{c} \beta\right)^{n} \leqq F(\beta, z) d V$ on $N$, then $F$ has an upper barrier on $\Omega$. 
We now state the main theorem.

THEOREM 3.4. Let $M$ be an $n$-dimensional Stein manifold with a volume form $d V$ and $\Omega$ a relatively compact, $C^{0}$ strictly pseudoconvex domain $M$. Let $F \in L_{\mathrm{loc}}^{\infty}(\boldsymbol{R} \times \Omega)$ with $F \geqq 0$ such that $F(t, z)$ is bounded on $\Omega$ for all fixed $t \in \boldsymbol{R}$, and continuous and nondecreasing in $t$ for every fixed $z \in \Omega$. If there exists an upper barrier for $F$ on $\Omega$, then

$$
u(z):=\sup \left\{v(z) ; v \in P(\Omega) \cap L_{\mathrm{loc}}^{\infty}(\Omega),\left(d d^{c} v\right)^{n} \geqq F(v, z) d V\right\}
$$

is a solution of the Dirichlet problem (3.1).

Proof. Let $\left\{\phi_{j}\right\}$ be a sequence of continuous functions on $\partial \Omega$ with $\phi_{1} \leqq \phi_{2} \leqq \cdots$ and $\lim _{j} \phi_{j}(z)=+\infty$ for $z \in \partial \Omega$. From the hypothesis about $F$, we see that the restriction of the function $F(t, z)$ to the set $\left(-\infty, \max \phi_{j}\right] \times \Omega$ is bounded for each $j$, so that by Theorem 3.1 there exists a unique solution $u$, to the Dirichlet problem for the data $\phi$, and $F$ for each $j$, i. e.,

$$
\begin{aligned}
& u_{j} \in P(\Omega) \cap L^{\infty}(\Omega), \\
& \lim _{\Omega \ni z \rightarrow \zeta} u_{j}(z)=\phi_{j}(\zeta) \quad \text { for } \quad \zeta \in \partial \Omega \text { and } \\
& \left(d d^{c} u_{j}\right)^{n}=F\left(u_{\jmath}, z\right) d V \quad \text { on } \Omega .
\end{aligned}
$$

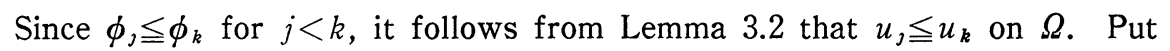

$$
\tilde{u}(z):=\left(\lim _{j} u_{j}(z)\right)^{*} \quad \text { for } \quad z \in \Omega \text {. }
$$

Clearly, $\tilde{u} \leqq u^{*}$ on $\Omega, \lim _{\Omega \ni z \rightarrow \zeta} \tilde{u}(z)=+\infty$ for every $\zeta \in \partial \Omega$ and the existence of the upper barrier implies that $\tilde{u}$ and $u^{*}$ are locally bounded plurisubharmonic functions on $\Omega$. Since $u_{j}(z)$ increases to $\tilde{u}(z)$ almost everywhere in $\Omega$, it holds from the continuity of $t \rightarrow F(t, z)$ on $R$ that $\left(d d^{c} \tilde{u}\right)^{n}=\lim _{j}\left(d d^{c} u_{j}\right)^{n}=\lim _{j} F\left(u_{j}, z\right) d V=$ $F(\tilde{u}, z) d V$. Therefore, $\tilde{u} \leqq u$ on $\Omega$, and $\tilde{u}$ is a solution of (3.1). Now, we can construct a solution, using the continuous dependence on the domain. Since $\Omega$ is strictly pseudoconvex, there exists an increasing sequence $\left\{\Omega_{k}\right\}$ of strictly pseudoconvex domains such that $\Omega_{1} \Subset \Omega_{2} \Subset \cdots \Subset \Omega$ and $\bigcup_{k} \Omega_{k}=\Omega$. Then from the way as above, we have a function $\tilde{u}_{k} \in P\left(\Omega_{k}\right) \cap L_{\mathrm{loc}}^{\infty}\left(\Omega_{k}\right)$ which satisfies

$$
\begin{aligned}
& \left(d d^{c} \tilde{u}_{k}\right)^{n}=F\left(u_{k}, z\right) d V \quad \text { on } \Omega_{k} \quad \text { and } \\
& \lim _{z \rightarrow \partial \Omega_{k}} \tilde{u}_{k}(z)=+\infty
\end{aligned}
$$

for each $k$. Since $\tilde{u}_{k+1}$ is bounded in $\Omega_{k}$, it follows from Lemma 3.2 that $\tilde{u}_{k+1} \leqq \tilde{u}_{k}$ on $\Omega_{k}$ for each $k$. From the same reason, we also have $v \leqq \tilde{u}_{k}$ on $\Omega_{k}$ for every $v \in P(\Omega) \cap L_{\text {loc }}^{\infty}(\Omega)$ with $\left(d d^{c} v\right)^{n} \geqq F(v, z) d V$ on $\Omega$ and each $k$. Thus if we put $\tilde{v}(z):=\lim _{k} \tilde{u}_{k}(z)$ for $z \in \Omega$, it is clear that $\tilde{v} \in P(\Omega) \cap L_{\text {loc }}^{\infty}(\Omega)$ and $u \leqq \tilde{v}$ on 
$\Omega$. Moreover, we see

$$
\begin{aligned}
\left(d d^{c} \tilde{v}\right)^{n} & =\lim _{k}\left(d d^{c} \tilde{u}_{k}\right)^{n}=\lim _{k} F\left(\tilde{u}_{k}, z\right) d V \\
& =F(\tilde{v}, z) d V \quad \text { on } \Omega .
\end{aligned}
$$

Hence, $\tilde{v} \leqq u$ on $\Omega$, so that $\tilde{v}=u$. Since $\tilde{u} \leqq u$ on $\Omega, \lim _{z \rightarrow \partial \Omega} u(z)=+\infty$, therefore $u$ is a solution of the Dirichlet problem (3.1).

Q.E.D.

Proof of Theorem in $\S 1$. Let $F$ be the function in Theorem 3.4 which satisfies $F(t, z) \geqq e^{k t}$ on $\boldsymbol{R} \times \Omega$ for some constant $k>0$. Then by Lemma 3.3, $F$ has an upper barrier on $\Omega$. In fact, on the ball $\Omega(\varepsilon):=\left\{z \in C^{n}:\|z\|<\varepsilon\right\}$,

$$
\beta(z):=\left[-(n+1) \log \left\{1-(\|z\| / \varepsilon)^{2}\right\}+n \log (n+1) / k \varepsilon^{2}+\log 4^{n} n !\right] / k
$$

is the solution of problem (3.1) with $M=C^{n}, \Omega=B(\varepsilon)$, and $F(t)=e^{k t}$. Thus by Theorem 3.4 if $\Omega$ is a strictly pseudoconvex, relatively compact domain in a Stein manifold, for the function $F$ as above we have a solution of problem (3.1).

Q.E.D.

\section{REFERENCES}

[1] Asaba, T., Asymptotic Dirichlet problem for a complex Monge-Ampère operator, Osaka J. Math. 23 (1986), 815-821.

[2] BEDFORD, E., The operator $\left(d d^{c}\right)^{n}$ on complex spaces, "Séminaire P. Lelong, H. Skoda 1980/81," Lecture Notes in Math., 919, Springer-Verlag, Berlin 1982, 294-323.

[3] Bedford, E. And TAYlor, B. A., The Dirichlet problem for a complex MongeAmpère equation, Invent. Math. 37 (1976), 1-44.

[4] BEDFord, E. AND TAYLOR, B. A., Variational properties of the complex MongeAmpère equation, I. Dirichlet principle, Duke Math. J. 45 (1978), 375-403.

[5] BEDFORD, E. AND TAYLOR, B. A., The Dirichlet problem for an equation of complex Monge-Ampère type, Partial Differential Equations and Geometry (ed. by C. Byrnes), Decker, (1979), 39-50.

[6] BEDFORD, E. AND TAYLOR, B. A., A new capacity for plurisubharmonic functions, Acta Math. 149 (1982), 1-41.

[7] BRemermann, H., On a generalized Dirichlet problem for plurisubharmonic functions and pseudoconvex domains. Characterization of Sỉlov boundaries, Trans. Amer. Math. Soc. 91 (1959), 246-276.

[8] Caffarelli, L., Kohn, J. J., Nirenberg, L. And Spruck, J., The Dirichlet problem for nonlinear second-order elliptic equations. II. Complex Monge-Ampère, and uniformly elliptic, equations, Comm. Pure Appl. Math. 38 (1985), 209-252.

[9] Cegrell, U., On the Dirichlet problem for the complex Monge-Ampère operator, Math. Z. 185 (1984), 247-251.

[10] ChenG, S. Y. AND YAU, S. T., On the existence of a Kähler metric on non-compact complex manifolds and the regularity of Fefferman's equation, Comm. Pure Appl. Math. 33 (1980), 507-544.

[11] Fukushima, M., A stochastic approach to the minimum principle for the complex 
Monge-Ampère operator, Proc. Conference on Stochastic Processes and their Applications, Lecture Notes in Math., 1203, Springer, 1985.

[12] Fukushima, M. ANd OKada, M., On Dirichlet forms for plurisubharmonic functions, Acta Math. 159 (1987), 171-213.

[13] KaneKo, H., A stochastic resolution of a complex Monge-Ampère equation on a negative curved Kähler manifold, Osaka J. Math. 24 (1987), 307-319.

[14] MoK, N. ANd YAU, S. T., Completeness of the Kähler-Einstein metric on bounded domains and the characterization of domains of holomorphy by curvature conditions, Proc. Symp. Pure Math. Amer. Math. Soc. 39 (1983), 41-59.

[15] LeLONG, P., Fonctions plurisousharmoniques et formes différentielles positives, Gordon and Breach, Paris, 1968.

[16] WaLsh, J.B., Continuity of envelopes of plurisubharmonic functions, J. Math. Mech., 18 (1968), 143-148.

TOKYo Institute of TECHNOLOGY

Department of Mathematics

MEGURO-KU, TOKYO, JAPAN 